\title{
Off-label pasireotide treatment in one insulinoma patient with an atypical presentation and intolerant to diazoxide
}

\author{
Federica Sileo ${ }^{1,2} \cdot$ Biagio Cangiano $^{1,2} \cdot$ Chiara Cacciatore $^{2} \cdot$ Jessica Amarù ${ }^{3}$. Federico Gatto ${ }^{4}$ Manuela Albertelli ${ }^{3}$. \\ Massimo Falconi ${ }^{5}$. Giovanni Vitale ${ }^{1,6} \cdot$ Luca Persani $\mathbb{1}^{1,2}$
}

Received: 29 April 2020 / Accepted: 24 June 2020 / Published online: 3 July 2020

(c) Springer Science+Business Media, LLC, part of Springer Nature 2020

Neuroendocrine Neoplasms (NENs) are rare, but their incidence is constantly increasing. Insulinoma is a functioning NEN, with an incidence of 1-4 cases/million/year [1]. Despite its benignity in $90 \%$ of cases [2], it is a dangerous condition due to hypoglycemic events and the intrinsic risks can be further extended in case of atypical presentation. Surgery is the treatment of choice for insulinoma, but biochemical diagnosis and localization of the lesion can be challenging, and patients may remain exposed to hypoglycemia risks for weeks. In the meantime, a medical control of the disease is required [2]. We present the off-label use of pasireotide for presurgical management of an atypical insulinoma.

A 42-year-old man had recurrent episodes of persistent diplopia for seven years. During one of these episodes, he was admitted to an emergency room revealing a profound hypoglycemia $(27 \mathrm{mg} / \mathrm{dL})$, with complete resolution of the symptoms after glucose administration. His past medical history was unremarkable, except for autoimmune hypothyroidism and removal of colon polyp. He was overweight

Luca Persani

luca.persani@unimi.it

1 Department of Clinical Sciences and Community Health, University of Milan, Milan, Italy

2 Lab of Endocrine and Metabolic Research, Department of Endocrine and Metabolic Diseases, IRCCS Istituto Auxologico Italiano, Milan, Italy

3 Endocrinology Unit, Department of Internal Medicine and Medical Specialties, University of Genoa, Genoa, Italy

4 Endocrinology Unit, Department of Specialist Medicine, IRCCS Ospedale Policlinico San Martino, Genoa, Italy

5 Division of Pancreatic Surgery, Pancreas Translational \& Clinical Research Center, Università Vita Salute, IRCCS San Raffaele Scientific Institute, Milan, Italy

6 Istituto Auxologico Italiano, IRCCS, Laboratorio Sperimentale di Ricerche di Neuroendocrinologia Geriatrica ed Oncologica, Cusano Milanino, Milan, Italy $\left(27 \mathrm{~kg} / \mathrm{m}^{2}\right)$, had negative anti-insulin and anti-GAD antibodies, but underwent an oral glucose tolerance test (OGTT) (glycemia: baseline 77; +120 min: $238 \mathrm{mg} / \mathrm{dL}$ ) leading to the diagnosis of diabetes mellitus type 2 (DM2) and reactive post-prandial hypoglycemia. He was put on a $1600 \mathrm{kcal}$ diet with morning and afternoon snacks and acarbose $50 \mathrm{mg}$ at principal meals. The patient lost $5 \mathrm{~kg}$ (BMI: $24.1 \mathrm{~kg} / \mathrm{m}^{2}$ ) but continued to register several fasting and postprandial hypoglycemic events always accompanied by diplopia. Thereafter, the patient came to our attention. Adrenal and pituitary deficiencies were excluded, and he was monitored for 5 hours after a new OGTT: this test resulted negative for diabetes, but a reactive hypoglycemia was registered $(+240 \mathrm{~min}: 38 \mathrm{mg} / \mathrm{dL})$. A fasting test revealed a persistent pauci-symptomatic hypoglycemia $(31 \mathrm{mg} / \mathrm{dL})$ with detectable values of insulin $(7.3 \mathrm{mU} / \mathrm{L})$ and C-peptide $(1.95 \mathrm{mcg} / \mathrm{L}) 29 \mathrm{~h}$ after the start. A diagnosis of insulin-dependent hypoglycemia was given, and an MRI showed a $1.5 \times 1.0 \mathrm{~cm}$ hypointense lesion (with a late-phase enhancement) in the pancreas head (Fig. 1a). An endoscopic ultrasound confirmed a solid hypoechoic lesion that was biopsied. At cytology, there were many isolated cells with atypical nuclei and positive immunostaining for insulin and chromogranin A $(\mathrm{Ki} 67=1 \%)$. The patient started a diazoxide treatment (3.0-5.7 mg/Kg). Despite the improvement of glycemic control with the higher dose (Fig. 1b), the patient experienced a worsening of dysgeusia, anorexia, nausea and a significant weight loss. Since surgery was planned several weeks later, an off-label treatment with pasireotide LAR ( $40 \mathrm{mg} / \mathrm{month})$ was initiated after informed consent. The patient experienced a significant improvement of glycemic control up to surgery (Fig. 1b, c). The treatment with pasireotide lasted 2 months with few well-tolerated episodes of diarrhea, then the patient underwent a duodenocephalopancreasectomy. For the following 3 years, the patient did not report any clinical manifestation of hypoglycemia. The repeated monitoring did not show any hypoglycemic event and an OGTT proved a normal glucose tolerance. 

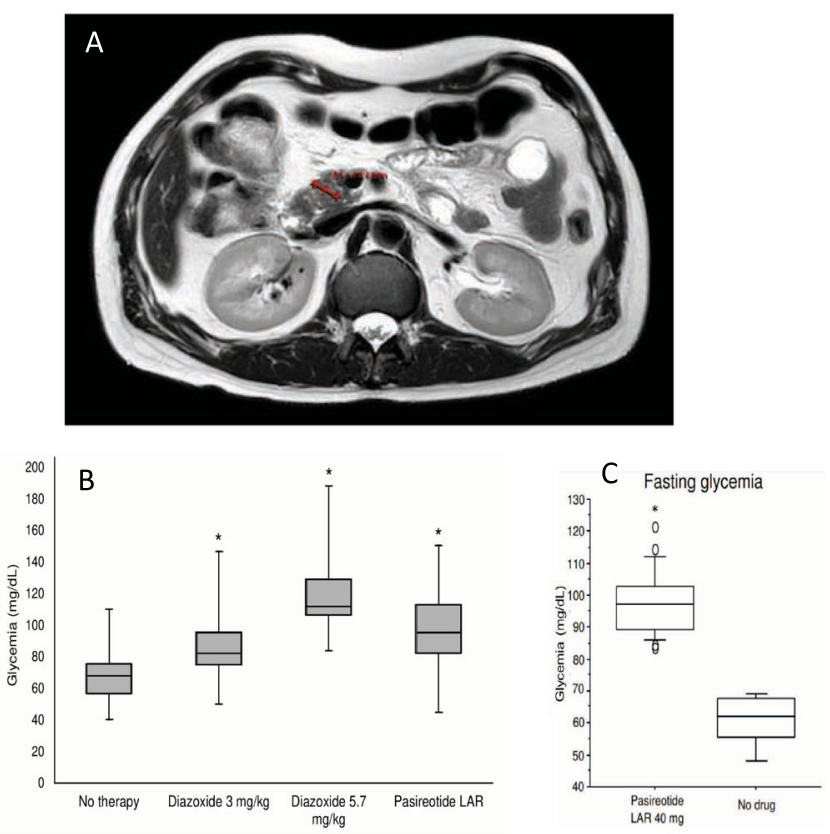

Fig. 1 a imaging of the lesion at MRI, the lesion of the head of the pancreas (measured with a red string), bilateral renal angiomyolipomas and an adenomyoma of the gallbladder; $\mathbf{b}$ Graph showing the difference of random capillary glycemia without therapy (first box plot), with diazoxide $3 \mathrm{mg} / \mathrm{kg}$ (second box plot), with diazoxide $5.7 \mathrm{mg} / \mathrm{kg}$ (third box plot) and with pasireotide (fourth box plot). The glycemic values registered during treatments were all significantly different from baseline at a M-Whitney comparison test $(* p<0.0001)$. Pasireotide LAR pasireotide long-acting release. c Box plots showing fasting glycemia levels at baseline and during Pasireotide LAR. The difference is highly significant at a M-Whitney comparison test $\left({ }^{*} p<\right.$ 0.0001); $\mathbf{d}$ immunohistochemical expression of $\mathrm{SST}_{1}$ and $\mathrm{SST}_{3}$. A, B:

The diagnosis of a pancreatic NEN was confirmed with an immunostaining positive for Cromogranin A and insulin at histology. Surprisingly, immunostaining for $\mathrm{SST}_{2}$ and $\mathrm{SST}_{5}$ resulted negative. On additional staining, $\mathrm{SST}_{1}$ revealed a moderate nuclear positivity, while $\mathrm{SST}_{3}$ expression showed a weak cytoplasmic staining. SST protein expression, evaluated by immunoreactivity scoring system (IRS) [3], showed a high-intermediate score $(\mathrm{IRS}=6)$ for $\mathrm{SST}_{1}$ and a low IRS (IRS =2) for $\mathrm{SST}_{3}$ (Fig. 1d).

Due to the young age of onset, the patient underwent genetic analysis for a MEN clinical variant on leukocyte DNA. A heterozygous variation (c.31C $>\mathrm{T})$ in exon 1 of $C D K N 1 b$ gene was discovered. This variation replaces a highly conserved proline at codon 11 of p27 protein (p. Pro11Ser). This variant is rare in population databases (rs779193240, ExAC: $0.008 \%$ ) and was previously reported in an individual with prostate cancer [4]. An analysis in silico $[5,6]$ showed a deleterious biological effect of p.P11S (Polyphen score: 0.995, range for damaging prediction (RDP): 0.85-1.0; SIFT score: 0; RDP: 0-0.05; REVEL score: 0.566; RDP > 0.5; MetalR score: 0.726; RDP: 0.5-1.0). In addition, the patient reported to have a first-degree cousin
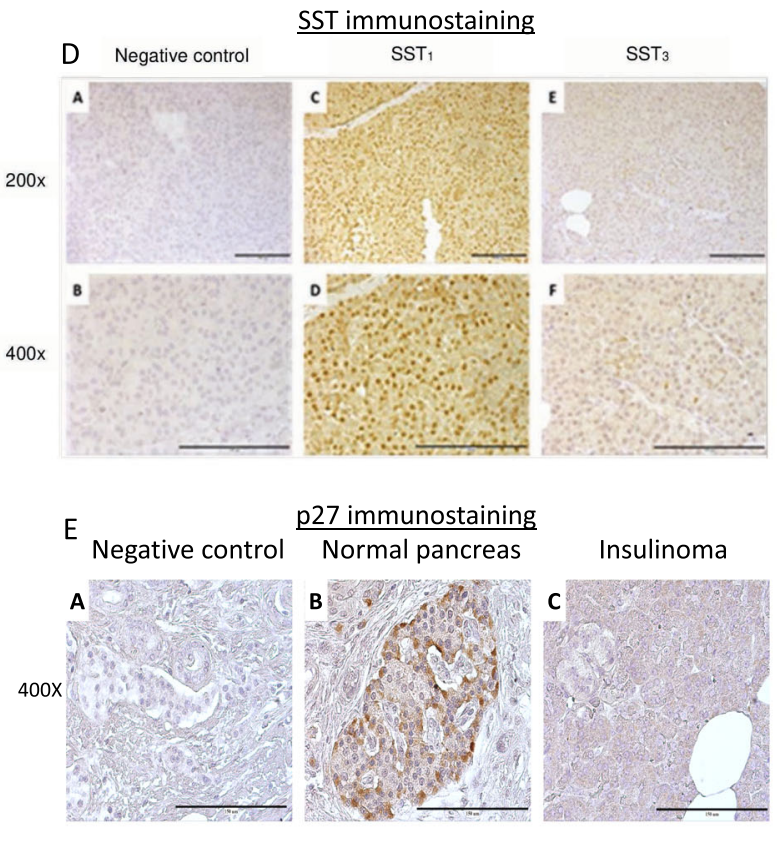

negative control (IRS $=0$ ); $\mathrm{C}, \mathrm{D}: \mathrm{SST}_{1}$ expression (polyclonal antibody, ab2366); E, F: $\mathrm{SST}_{3}$ expression. (polyclonal antibody, ab28680); Scale bar $150 \mu \mathrm{m}$; magnification: (A, C, E) 200×, (B, D, F) 400x; e Immunohistochemical expression of p27 (anti p27-KIP1 [Y236] monoclonal antibody, ab32034). A normal pancreatic tissue with omission of primary antibody; B p27 expression in normal pancreatic tissue; C p27 expression in the insulinoma tissue. The absent p27 immunostaining in the insulinoma of this patient gives definitive support to the pathogenic role of the variant, associated with the loss of expression of wild-type p27 allele in the tumor sample. Scale bar $150 \mu \mathrm{m}$; magnification, 400x. All antibodies were purchased from Abcam (Cambridge, UK)

with a recent diagnosis of pancreatic NET, and she was also found to be carrier of the p.P11S variant, whereas the $C D K N 1 b$ gene was wild-type in other four unaffected family members (age: $25-73$ years). The absent p27 immunostaining in the insulinoma of this patient (Fig. 1e) gives definitive support to the pathogenic role of the variant.

In this patient, hypoglycemic events had mild clinical manifestations (diplopia) most probably due to the long history of the disease and the progressive adaptation thanks to the efficiency of counterregulatory mechanisms. The diagnostic delay (about 8 years) of this atypical insulinoma was further extended by the results of the first OGTT indicating a DM2. The loss of $5 \mathrm{~kg}$ body weight was however sufficient to recover a normal glucose tolerance at succeeding OGTTs.

Once reached the diagnosis, the medical treatment was given for about 10 weeks in order to avoid the additional hypoglycemic events exposing the patient and his relatives to the potential risks of an unpredictable symptomatic hypoglycemia. Unfortunately, the patient was intolerant to diazoxide and we sought for alternative medical treatment. Taking into account the benign features of this lesion, we 
first considered classic somatostatin analogues (SSAs). However, these drugs are reported to be effective in $50 \%$ of cases and to be able to cause paradoxical hypoglycemic effects [7]. On the other hand, Pasireotide is a secondgeneration SSA with a preserved affinity for $\mathrm{SST}_{2}$ but considerable affinity for $\mathrm{SST}_{5}$ and for $\mathrm{SST}_{1}, \mathrm{SST}_{3}$, as well. It is approved for treatment of Cushing's disease and acromegaly [8] but its use in insulinomas was previously reported in only two cases of malignant insulinomas $[9,10]$. Hyperglycemia is one of the most significant side effects of this drug, with a prevalence of $79 \%$ [11], and it is more likely to occur in patients with underlying diabetes or prediabetes [12]. Such Pasireotide characteristics were of particular advantage in this patient with a history of DM2. Accordingly, Pasireotide achieved a drastic reduction of hypoglycemic events.

A recent study from Henry et al. [13] tried to define the mechanisms through which Pasireotide causes hyperglycemia in healthy volunteers: results showed an inhibition of insulin, a more modest suppressive effect on glucagon and a reduced incretin response (GLP-1 and GIP). In this insulinoma, we found a strong expression of $\mathrm{SST}_{1}$ which could perhaps explain the response to the drug, but its main nuclear localization leaves some doubts. A similar situation is found in another patient, whose malignant insulinoma, negative for $\mathrm{SST}_{5}$ and $\mathrm{SST}_{3}$ and positive for $\mathrm{SST}_{2}$, responded to treatment with Pasireotide but surprisingly not with Lanreotide [10]. While the effects on insulin and glucagon could be explained by the binding of Pasireotide to SSTs, the suppressed incretin secretion would instead be mediated by not yet identified mechanisms. Such alternative mechanism of action could perhaps explain the efficient control of hypoglycemia by Pasireotide in this insulinoma with inconclusive findings at immunostaining. Nevertheless, a downregulation of $\mathrm{SST}_{2}$ and $\mathrm{SST}_{5}$ induced by the SSA treatment cannot be excluded [14]. Still, these data prompt further studies aimed to investigate all the interactions of Pasireotide with yet unknown intermediaries and their effect on glucose metabolism.

In conclusion, we describe a man with longstanding atypical hypoglycemic manifestations who, after an initial diagnosis of DM2, was found to be affected with a pancreatic insulinoma associated with a rare deleterious $C D K N 1 b$ variant. The efficient control of hypoglycemia by pasireotide LAR in an insulinoma with low $\mathrm{SST}_{2} / \mathrm{SST}_{5}$ expression opens novel perspectives on the mechanisms underlying pasireotide action on glucose metabolism.

\section{Data availability}

The datasets generated during and/or analyzed during the current study are available from the corresponding author on reasonable request.
Funding This work was partially supported by Ricerca Corrente funds of IRCCS Istituto Auxologico Italiano (code: 02C502_2005).

Author contributions The study was conceived and designed by $\mathrm{BC}$, MF, GV and LP. Immunohistochemistry was performed by JA and FG. Data collection and analysis were performed by FS, BC, FG and MA. The first draft of the manuscript was written by FS; all authors revised the draft and the manuscript was finalized by LP. All authors read and approved the final version of the manuscript.

\section{Compliance with ethical standards}

Conflict of interest The authors declare that they have no conflict of interest.

Consent for publication Patient signed informed consent to publish the data.

Consent to participate The patient signed informed consents to the off-label treatment with pasireotide and to genetic studies.

Ethics approval The study was approved by the institutional review board of Istituto Auxologico Italiano, IRCCS and performed in accordance with the ethical standards as laid down in the 1964 Declaration of Helsinki and its later amendments or comparable ethical standards (Date: January 2005/No. 02C502_2005).

Publisher's note Springer Nature remains neutral with regard to jurisdictional claims in published maps and institutional affiliations.

\section{References}

1. F.J. Service, M.M. McMahon, P.C. O'Brien, D.J. Ballard, Functioning insulinoma-incidence, recurrence, and long-term survival of patients: a 60-year study. Mayo Clin. Proc. 66(7), 711-719 (1991)

2. M. Falconi, B. Eriksson, G. Kaltsas, D.K. Bartsch, J. Capdevila, M. Caplin, B. Kos-Kudla, D. Kwekkeboom, G. Rindi, G. KlÖppel, N. Reed, R. Kianmanesh, R.T. Jensen, ENETS consensus guidelines update for the management of patients with functional pancreatic neuroendocrine tumors and non-functional pancreatic neuroendocrine tumors. Neuroendocrinology 103, 153-171 (2016)

3. F. Gatto, R.A. Feelders, R. Van Der Pas, J.M. Kros, M. Waaijers, D. Sprij-Mooij, S.J.C.M.M. Neggers, A.J. Van Der Lelij, F. Minuto, S.W.J. Lamberts, W.W. De Herder, D. Ferone, L.J. Hofland, Immunoreactivity score using an anti-sst2A receptor monoclonal antibody strongly predicts the biochemical response to adjuvant treatment with somatostatin analogs in acromegaly. J. Clin. Endocrinol. Metab. 98(1), E66-E71 (2013)

4. C. Cybulski, B. Gliniewicz, A. Sikorski, J. Kładny, T. Huzarski, J. Gronwald, T. Byrski, T. Debniak, B. Gorski, A. Jakubowska, D. Wokolorczyk, S.A. Narod, J. Lubiñski, Epistatic relationship between the cancer susceptibility genes CHEK2 and p27. Cancer Epidemiol. Biomarkers Prev. 16(3), 572-576 (2007)

5. L. Persani, T. de Filippis, C. Colombo, D. Gentilini, GENETICS IN ENDOCRINOLOGY: genetic diagnosis of endocrine diseases by NGS: novel scenarios and unpredictable results and risks. Eur. J. Endocrinol. 179(3), R111-R123 (2018)

6. T. de Filippis, G. Gelmini, E. Paraboschi, M.C. Vigone, M. Di Frenna, F. Marelli, M. Bonomi, A. Cassio, D. Larizza, M. Moro, G. Radetti, M. Salerno, D. Ardissino, G. Weber, D. Gentilini, F. Guizzardi, S. Duga, L. Persani, A frequent oligogenic 
involvement in congenital hypothyroidism. Hum. Mol. Genet. 26 (13), 2507-2514 (2017)

7. Vezzosi D., Bennet A., Rochaix P., Courbon F., Selves J., Pradere B., Buscail L., Susini C., Caron P. Octreotide in insulinoma patients: efficacy on hypoglycemia, relationships with Octreoscan scintigraphy and immunostaining with anti-sst2A and anti-sst5 antibodies. Eur. J. Endocrinol. 2005. https://doi.org/10.1530/eje.1. 01901

8. C. Bruns, I. Lewis, U. Briner, G. Meno-Tetang, G. Weckbecker, SOM230: a novel somatostatin peptidomimetic with broad somatotropin release inhibiting factor (SRIF) receptor binding and a unique antisecretory profile. Eur. J. Endocrinol. 146(5), 707-716 (2002)

9. A. Tirosh, S.M. Stemmer, E. Solomonov, E. Elnekave, W. Saeger, Y. Ravkin, K. Nir, Y. Talmor, I. Shimon, Pasireotide for malignant insulinoma. Hormones. 15(2), 271-276 (2016)

10. N.S. Hendren, K. Panach, T.J. Brown, L. Peng, M.S. Beg, J. Weissler, S. Mirfakhraee, Pasireotide for the treatment of refractory hypoglycaemia from malignant insulinoma. Clin. Endocrinol. (Oxf.) 88(2), 341-343 (2018)
11. M. Cives, P.L. Kunz, B. Morse, D. Coppola, M.J. Schell, T. Campos, P.T. Nguyen, P. Nandoskar, V. Khandelwal, J.R. Strosberg, Phase II clinical trial of pasireotide long-acting repeatable in patients with metastatic neuroendocrine tumors. Endocr. Relat. Cancer 22(1), 1-9 (2015)

12. L.K. Kvols, K.E. Oberg, T.M. O'Dorisio, P. Mohideen, W.W. de Herder, R. Arnold, K. Hu, Y. Zhang, G. Hughes, L. Anthony, B. Wiedenmann, Pasireotide (SOM230) shows efficacy and tolerability in the treatment of patients with advanced neuroendocrine tumors refractory or resistant to octreotide LAR: results from a phase II study. Endocr. Relat. Cancer 19(5), 657-666 (2012)

13. R.R. Henry, T.P. Ciaraldi, D. Armstrong, P. Burke, M. LiguerosSaylan, S. Mudaliar, Hyperglycemia associated with pasireotide: results from a mechanistic study in healthy volunteers. J. Clin. Endocrinol. Metab. 98(8), 3446-3453 (2013)

14. M. Li, W. Li, H.J. Kim, Q. Yao, C. Chen, W.E. Fisher, Characterization of somatostatin receptor expression in human pancreatic cancer using real-time RT-PCR. J. Surg. Res. 119, 130-137 (2004) 